\title{
Total pericystectomy in liver echynococcal cysts using bipolar sealing coupled with saline device
}

\begin{abstract}
Aim: To evaluate the safety of bipolar sealing coupled with saline device, offering transcollation technology, in the radical procedure of total pericystectomy in liver echinococcal cysts.

Patients and Methods: 15 patients with liver echynococcal cysts underwent total pericystectomy using Bipolar Sealing Coupled with Saline Device. Additionally, two patients were treated with major hepatic excision, but these patients were not included because those cases were beyond the scope of this evaluation. Our main goal was to evaluate the safety of the procedure regarding perioperative bleeding, postoperative biliary leakage and abscess formation. Our secondary goal was to observe echinococcal cyst relapse after a period of 10 to 36 month follow up.
\end{abstract}

Results: All fifteen patients underwent total pericystectomy of liver echinococcal cysts, eleven with open surgery and two laparoscopically. Resection time was dependent upon the number, complexity and location of the cysts. Blood loss was minimal and blood transfusion was not required, neither intraoperatively nor postoperatively. Mortality was zero. No postoperative bile leakage or abscess formation was noted. No echinococcal cyst relapse was observed.

Conclusion: Total pericystectomy in liver echinococcal cysts using bipolar sealing coupled with saline device can be considered a safe radical technique, as it allows completion of the procedure with minimal blood loss. Further studies are needed to confirm our preliminary results.

Keywords: hydatid disease, cystic echinococcosis, liver, transcollation, aquamantys ${ }^{\circledR}$
Volume 3 Issue 6 - 2017

\author{
Parpounas C,' Morris D, ${ }^{2}$ Bramis K, ${ }^{3}$ \\ Papalambros A, ${ }^{4}$ Lazarou Vasiliki, ${ }^{\prime}$ \\ Charalambous C,' Petrou $A^{\prime}$ \\ 'Department of Surgery, University of Nicosia, Cyprus \\ ${ }^{2}$ Lerner Research Institute, Cleveland Clinic Foundation, USA \\ ${ }^{3}$ Senior Clinical Fellow in HPB Surgery and Liver Transplantation, \\ Royal Free Hospital, UK \\ ${ }^{4}$ Department of Surgery, University of Athens Medical School, \\ Greece
}

\begin{abstract}
Correspondence: Parpounas Constantinos MD, MA, Honorary Tutor of Surgery, St George's University of London Programme, University of Nicosia, 93 Agiou Nikolaou Street, Engomi, 2408 Nicosia, Senior Resident in General Surgery, New Nicosia General Hospital, Department of Surgery/Div HPB, Limassol Old Road No. 215, 2029 Nicosia, Cyprus, Tel +35799339889, Email parpounas@gmail.com
\end{abstract}

Received: November 09, 2017| Published: December 04, 2017

\section{Introduction}

In multiple and complicated liver echinococcal cysts, management is challenging and requires a combination of preoperative chemotherapy and surgical intervention. The optimal operative method in echinococcal cyst management is yet to be agreed, ${ }^{1,2}$ however it has been shown that radical procedures, including total pericystectomy, appear with fewer relapses and, the past years, there has been a shift towards the more aggressive surgical approach, due to the decreased postoperative complications and lower mortality when related to conservative techniques..$^{3-5}$ The main goal when treating echinococcal cysts is to prepare the patient properly, both preoperatively, by providing the right chemotherapeutic agent, and perform a safe surgical procedure, by avoiding rupture and spillage of the cyst, performing a virtually bloodless operation and keeping mortality and morbidity to minimum.

Bipolar sealing coupled with saline is an FDA approved approach for reducing intraoperative blood loss. It offers transcollation technology that couples radiofrequency energy with saline to provide haemostatic sealing of soft tissue. In this paper we present our experience with the device during total pericystectomy of echinococcal liver cysts. Our main goal was to evaluate the safety of the procedure regarding perioperative bleeding, postoperative biliary leakage and abscess formation. Our secondary goal was to observe echinococcal cyst relapse after a period of 10 to 36 month follow up.

\section{Patients and methods}

Between November 2011 and December 2016, 15 patients with liver echinococcal cysts underwent total pericystectomy at Nicosia General Hospital, Department of Surgery, Division of Hepatobiliary and Pancreas Surgery, using bipolar sealing coupled with saline device. There were nine female $(60,0 \%)$ and six male $(40,0 \%)$ patients with mean age of 43(range 23-74). The patients underwent preoperative assessment with clinical examination, abdominal ultrasound, computed tomography and serologic tests to establish the diagnosis. Six patients had one cyst, five patients had two cysts, two patients had three cysts and two had four cysts (Table 1). Most cysts were located at IV liver segment (seven cysts), followed by II and VII (six cysts each), then III and VI (four cysts each), then V (two cysts) and lastly VIII (one cyst) (Table 2). Seven cysts were less than $4 \mathrm{~cm}$ in diameter, 15 cysts were between $4-8 \mathrm{~cm}$, six cysts were $8-16 \mathrm{~cm}$ and two cysts were more than $16 \mathrm{~cm}$ (Table 3 ).

Table INumber of Echinococcal Cysts in Liver

\begin{tabular}{lllll}
\hline Number of echinococcal cysts in liver \\
\hline Number of Cysts & I & 2 & 3 & 4 \\
Number of Patients & 6 & 5 & 2 & 2 \\
\hline
\end{tabular}

Table 2 Location of Echinococcal Cysts in Liver

Location of echinococcal cysts in liver

\begin{tabular}{llllllll} 
Liver segment & IV & II & VII & III & VI & V & VIII \\
Number of Cysts & 7 & 6 & 6 & 4 & 4 & 2 & I \\
\hline
\end{tabular}


Table 3 Size of Echinococcal Cysts in Liver

\begin{tabular}{|c|c|c|c|c|}
\hline \multicolumn{5}{|c|}{ Size of echinococcal cysts in liver } \\
\hline Size of Cysts $(\mathrm{cm})$ & $<4$ & 8-Apr & 16-Aug & $>16$ \\
\hline Number of Cysts & 7 & 15 & 6 & 2 \\
\hline
\end{tabular}

Albendazole $400 \mathrm{mg}$ was prescribed in all patients twice per day for 28days before the surgery. Antibiotic prophylaxis (Cefuroxime $1.5 \mathrm{~g}$ and metronidazole $500 \mathrm{mg}$ ) was provided immediately prior to surgery. Bilateral subcostal or $\mathrm{j}$-incision was performed, and the usual abdominal exploration was taken place. The liver was mobilized, and gauzes soaked in hypertonic saline were used, isolating the cyst from the surrounding area. Intraoperative ultrasound of the liver was performed to exclude the presence of major intrahepatic vessels near the cysts. Total pericystectomy was then performed using the bipolar sealing coupled with saline device (Aquamantys ${ }^{\circledR}$ System). The bipolar sealing coupled with saline device was assembled and set with the presence of a manufacturer representative. The generator was connected to the Aquamantys ${ }^{\circledR}$ 9.5 XL Bipolar sealer (Medtronic Advanced Energy) when open surgery was going to be performed and to the Endo DBS 8.7 (Medtronic Advanced Energy) when the surgery was aimed to be performed laparoscopically. The device was set at $170 \mathrm{~W}$ with medium flow rate of $22 \mathrm{ml} / \mathrm{mm}$ based on manufacturer's recommendation. The total pericystectomy was performed by applying the tip of the device at the resection site with painting motion and spot-treating bleeding vessels, using constant suction to clear the saline applied for irrigation. Using this technique, it allowed structures up to $6 \mathrm{~mm}$ in diameter to be sealed without producing temperatures above 100C. Structures with diameter more than $6 \mathrm{~mm}$ were ligated with clips or ties. Pringle maneuver was not performed on any patient. After the completion of the procedure, intraoperative cholangiography or Propofol infusion test was performed where indicated to exclude bile leakage. In addition to the above cases, two patients had to undergo major hepatic resection due to the size, number and/or complexity of the liver echinococcal cysts. One of the two patients was presented with a large complicated cyst at the right lobe of the liver, perforating the diaphragm and producing a hepatopleural fistula. Right extended hepatectomy together with partial diaphragmatic excision and decortication was the treatment of choice. The second patient was presented with a large and complex cyst on the left lobe. Initially, unroofing of the cyst was performed and left hepatectomy was carried out. Postoperatively the patients were treated with analgesia, thromboprophylaxis and accordingly with gastroprophylaxis. After discharge the patients had a follow up of 10-36months.

\section{Results}

Open surgery was performed in 13 patients and laparoscopic surgery in two patients. The laparoscopic surgery was performed in patients with simple cysts, located at the periphery that was technically simple and safe. In all patients the bipolar sealing coupled with saline device was used. Resection time was dependent upon the number; complexity and location of the cysts with mean resection time of 38 minutes (range 24-96min). Blood loss was minimal in all operations and blood transfusion was not required, neither intraoperatively nor postoperatively. Mortality was zero. Fluid collection or abscess formation was not observed in any patient. Discharge was given after bowel return and control of pain with oral medication. The mean postoperative stay was five days (range 3-12days). The patients were then observed clinically after two and four weeks following the discharge. After the initial postoperative outpatient assessment, the patients were asked to return for further observation in three-six-ninemonth interval and then yearly. Clinical examination and serologic tests were performed in every visit. Radiologic examination (CT scan) was performed a year after the operation. Additional radiologic examinations (either US or CT scan) prior or after the first year were performed when indicated. In a period of 10-36month follow up, no echinococcal cyst relapse was no

\section{Discussion}

Total pericystectomy is a radical procedure in treating liver echinococcal cysts that is proved to offer fewer relapses when compared to more conservative techniques but it may be hazardous in complicated cysts. ${ }^{6}$ In this paper we describe our experience in using bipolar sealing coupled with saline device to manage parenchymal haemostasis in total pericystectomy. In liver surgery perioperative blood loss and possible perioperative or postoperative blood transfusion is of major concern due to increased mortality and morbidity that affect long term survival. Many authors tried to investigate methods in reducing blood loss during liver resection using different techniques and devices such as Cavitron Ultrasonic Surgical Aspiration, Water Jet, Harmonic scalpel, Ligasure, Gyrus, Tissuelink, Stapler, Habib technique and others. None of the above proved to be substantially superior over the other. What seems to be of major importance is the surgeon's preference related to his experience. ${ }^{7-10}$

Bipolar sealing coupled with saline is an FDA approved approach for reducing intraoperative blood loss. It offers transcollation technology that couples radiofrequency energy with saline to provide haemostatic sealing of soft tissue. It offers controlled thermal energy to the tissue, not exceeding the $100^{\circ} \mathrm{C}$, thanks to the continuous irrigation system and, in addition, it does not cause smoke or char formation during the process. The highly vascularized liver parenchyma is coagulated by the thermal effect of the system and causing contraction of Type I and Type II collagen found within the vessel walls. Structures between $3-6 \mathrm{~mm}$ in diameter can be safely sealed while the bigger ones can be isolated and either clipped or tied. From our experience and according to other authors, ${ }^{11}$ bipolar sealing coupled with saline device can achieve liver resection with minimal blood loss and without bile leakage.

Of course, there are some concerns when using bipolar sealing coupled with saline that must be addressed. Firstly, it offers a slow pace during liver transection, and in addition, it can sometimes prohibit the identification of main blood vessels or hepatic ducts, causing damage. Hence it should be used cautiously when working near these structures.

\section{Conclusion}

Total pericystectomy in liver echinococcal cysts using bipolar sealing coupled with saline can be considered a safe radical technique. It proved to allow completion of the procedure with minimal blood loss and without bile leakage in neither of our cases. Nevertheless, further studies are needed to confirm our preliminary results mainly by comparing this technology with other techniques that have already been mentioned above.

\section{Acknowledgements}

\section{None.}




\section{Conflicts of interest}

The authors declare that there is no conflict of interest regarding the publication of this paper.

\section{References}

1. Barnes SA, Lillemoe KD. Liver abscess and hydatid cyst disease. In: Zinner MJ, Schwartz SI, editors. Maingot's Abdominal Operation. 10th ed. USA: McGraw-Hill Professional; 1997.

2. Kammerer WS, Schantz PM. Echinococcal disease. Infect Dis Clin North Am. 1993;7(3):605-618.

3. Gollackner B, Längle F, Auer H, et al. Radical surgical therapy of abdominal cystic hydatid disease: factors of recurrence. World J Surg. 2000;24(6):717-721.

4. Cirenei A, Bertoldi I. Evolution of surgery for liver hydatidosis from 1950 to today: analysis of a personal experience. World J Surg. 2001;25(1):87-92.

5. Malik A, Bari UL, Amin R, et al. Surgical management of complicated hydatid cysts of the liver. World J Gastrointest Surg. 2010;2(3):78-84.
6. Gupta N, Javed A, Puri S, et al. Hepatic Hydatid: PAIR, Drain or Resect? J Gastrointest Surg. 2011;15(10):1829-1836.

7. Poon RTP. Current techniques of liver transection. HPB (Oxford). 2007;9(3):166-173.

8. Felekouras E, Petrou A, Neofytou K, et al. Combined ultrasonic aspiration and saline-linked radiofrequency precoagulation: a step toward bloodless liver resection without the need of liver inflow occlusion: analysis of 313 consecutive patients. World J Surg Oncol. 2014;12:357.

9. Moggia E, Rouse B, Simillis C, et al. Methods to decrease blood loss during liver resection: a network meta-analysis. Cochrane Database Syst Rev. 2016;10:CD010683.

10. Otsuka Y, Kaneko H, Cleary SP, et al. What is the best technique in parenchymal transection in laparoscopic liver resection? Comprehensive review for the clinical question on the 2nd International Consensus Conference on Laparoscopic Liver Resection. J Hepatobiliary Pancreat Sci. 2015;22(5):363-370.

11. Geller DA, Tsung A, Maheshwari V, et al. Hepatic resection in 170 patients using saline-cooled radiofrequency coagulation. HPB (Oxford). 2005;7(3):208-213. 